\title{
Nanomodulated electron beams via electron diffraction and emittance exchange for coherent x-ray generation
}

\author{
E. A. Nanni, ${ }^{1,2}$ W. S. Graves, ${ }^{3}$ and D. E. Moncton ${ }^{2}$ \\ ${ }^{1}$ SLAC National Accelerator Laboratory, Menlo Park, California 94025, USA \\ ${ }^{2}$ Massachusetts Institute of Technology, Cambridge, Massachusetts 02139, USA \\ ${ }^{3}$ Arizona State University, Tempe, Arizona 85287, USA
}

(Received 24 September 2017; published 19 January 2018)

\begin{abstract}
We present a new method for generation of relativistic electron beams with current modulation on the nanometer scale and below. The current modulation is produced by diffracting relativistic electrons in single crystal $\mathrm{Si}$, accelerating the diffracted beam and imaging the crystal structure, then transferring the image into the temporal dimension via emittance exchange. The modulation period can be tuned by adjusting electron optics after diffraction. This tunable longitudinal modulation can have a period as short as a few angstroms, enabling production of coherent hard x-rays from a source based on inverse Compton scattering with total accelerator length of approximately ten meters. Electron beam simulations from cathode emission through diffraction, acceleration, and image formation with variable magnification are presented along with estimates of the coherent x-ray output properties.
\end{abstract}

DOI: 10.1103/PhysRevAccelBeams.21.014401

\section{INTRODUCTION}

Hard x-ray free-electron lasers (FELs) such as LCLS, SACLA, and XFEL [1-3] rely on long linear accelerators to produce high energy $(>7 \mathrm{GeV}$ ) electron beams that meet the resonant condition for the x-ray wavelength $\lambda_{x} \sim \lambda_{u} / \gamma^{2}$ for output from an undulator with period $\lambda_{u}$ of a few centimeters and kinetic energy $E_{k} \approx \gamma m c^{2}$. The high electron energy required has the advantages of lowered electron beam geometric emittance becoming less than the diffraction-limited mode area of the coherent x-ray beam, and reduced space charge forces that might interfere with FEL gain. The drawbacks are that the required facilities are large and expensive with just a few contemplated around the world, and that the electron modulation and the resulting $\mathrm{x}$-ray beams produced by self-amplified spontaneous emission (SASE) are not fully coherent. FEL facilities are investigating several schemes in order to achieve greater temporal coherence including self-seeding [4-6], high-gain harmonic generation [7-9] and echoenhanced harmonic generation [10,11]. With harmonic generation, a seed laser at a longer wavelength is used to initiate the modulation on the electron bunch which then radiates at a harmonic of the laser wavelength with results demonstrated at extreme UV and soft x-ray wavelengths.

Published by the American Physical Society under the terms of the Creative Commons Attribution 4.0 International license. Further distribution of this work must maintain attribution to the author(s) and the published article's title, journal citation, and DOI.
Self-seeding uses a small portion of the SASE spectrum to seed a coherent pulse in a subsequent undulator. These techniques continue to require the use of $\mathrm{GeV}$ electron beams, include the addition of undulators and dispersive sections; and have proved challenging to scale to hard $\mathrm{x}$-ray wavelengths.

Transmission electron microscopes (TEMs) meanwhile produce a coherent spatial electron modulation at the scale of angstroms and do so using electrons with much lower energies of a few hundred $\mathrm{keV}$ from a compact device. This demonstrates that an ultrarelativistic beam is not a priori necessary for the angstrom-scale electron modulation typically produced by an x-ray FEL. Furthermore, this modulation can have a much greater coherence. We take advantage of TEM-like electron diffraction of a modestly relativistic $7 \mathrm{MeV}$ electron beam from a silicon target in combination with a previous concept $[12,13]$ for transforming a spatial modulation to a coherent temporal modulation at short wavelength to produce an electron beam suitable for coherent x-ray generation. Our earlier work relied on generation of an electron beam from a nanostructured cathode that was limited to scales of hundreds of nanometers or longer. The current concept has several important advantages including potential for hard x-rays set by the atomic-scale limits of the diffracting crystal, mitigation of space charge effects due to diffraction at relativistic energy and use of a robust conventional flat cathode to produce the electrons.

The target that will be used to produce a transverse density modulation in the electron bunch is a silicon grating, with the grating pattern approximately normal to 


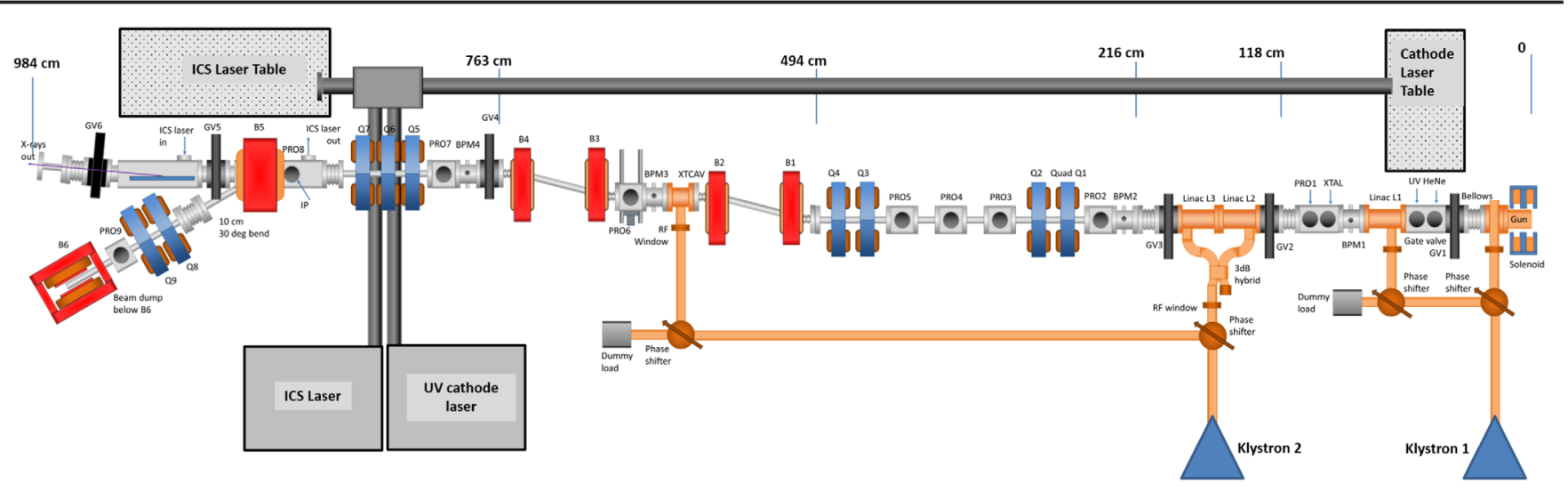

FIG. 1. Schematic of the compact coherent X-ray source with rf photo-injector, electron diffraction crystal, X-band linac, EEX line and ICS laser interaction area. Entire assembly is approximately $10 \mathrm{~m}$ long.

the electron beam's direction of propagation. As shown in Sec. III, the varying thickness of the silicon results in a spatially varying modulation of the probability for the electron to be diffracted by a particular Bragg peak in the basic silicon structure. The thickness parameters of the grating can be chosen to optimize this diffraction contrast using the effect of dynamical extinction. After the target, either the Bragg-scattered electrons or forward-scattered electrons are accelerated and sent through the imaging optics. With this diffraction-contrast imaging, the limitation on the modulation period set at the grating is the minimum feature size that can be fabricated, of order $100 \mathrm{~nm}$ for standard lithographic techniques. However, this modulation period can be magnified or demagnified downstream with standard magnetic optics. Here we show that it is possible to demagnify the modulation period by a factor of 150 producing modulation periods of one $\mathrm{nm}$. This approach can be extended to modulation at the Angstrom scale, as discussed in Sec. VI, with a uniform target thickness and the interference of multiple diffracted beams, similar to phase-contrast imaging used in high-voltage electron microscopy.

A hard x-ray FEL based on this technology would fit comfortably in existing industrial, academic and medical laboratories at a cost comparable to other sophisticated analytical instruments. A schematic of the coherent x-ray source is shown in Fig. 1 with a total length of approximately $10 \mathrm{~m}$. We estimate that the output pulse for this source will have about $10 \mathrm{~nJ}$ of energy compared to the millijoule levels of the large machines. However, it will be fully coherent, unlike SASE, and would be an excellent source for seeding temporally coherent $\mathrm{x}$-rays from the large machines as well as directly producing new science opportunities for a broad range of applications.

\section{ELECTRON DIFFRACTION}

Ultrafast electron diffraction experiments using electron bunches produced by a rf photo-injector with an electron energy on the order of a few $\mathrm{MeV}$ and a charge of several pC [14-18] have demonstrated the feasibility of obtaining useful diffraction patterns with low emittance and high peak current electron beams. The main constraint for the use of a rf photo-injector for electron diffraction is the ability to achieve a low enough emittance or angular spread of the incoming electron bunch. Additionally, the diffraction or phase contrast image must be preserved through the imaging optics.

In the present case we model a $1 \mathrm{pC}$ electron bunch photoemitted from a flat cathode in a 3.5 cell rf gun operating at $9.3 \mathrm{GHz}$ with a peak cathode field of $170 \mathrm{MV} / \mathrm{m}$ and $\mathrm{rf}$ phase of $80^{\circ}$ at emission with PARMELA [19]. The electron bunch is generated by a UV laser pulse with $30 \mathrm{fs}$ full width and a parabolic spatial distribution with RMS size of $30 \mu \mathrm{m}$ in order to produce a 3-dimensional ellipsoid in blow-out mode [20,21]. The normalized emittance is

$$
\varepsilon_{x n}=\frac{1}{m_{e} c} \sqrt{\left\langle x^{2}\right\rangle\left\langle p_{x}^{2}\right\rangle-\left\langle x p_{x}\right\rangle^{2}}
$$

where $x$ is the transverse coordinate, $p_{x}$ is the transverse momentum, $m_{e}$ is the electron mass and $c$ is the speed of light, with an initial value $\varepsilon_{x n}=9 \mathrm{~nm}-\mathrm{rad}$, which is an aggressive assumption but is supported by recent measurements [22]. The gun exit energy is $4.5 \mathrm{MeV}$. A short $20 \mathrm{~cm}$ linac then accelerates the beam to $7 \mathrm{MeV}$ and removes its time-energy chirp. A solenoid magnet surrounding the gun collimates the electron beam resulting in an RMS spot size at the crystal of $\sigma_{x}=101 \mu \mathrm{m}$ with an angular distribution $\sigma_{x^{\prime}}=7.7 \mu \mathrm{rad}$ which is more than one order of magnitude smaller than the Bragg angle. Simulation results show bunch length expansion to an RMS length of $100 \mathrm{fs}$ with a peak current of $3.2 \mathrm{~A}$.

Prior to analyzing the formation of a transverse electrondensity modulation, we describe the underlying physics of electron diffraction occurring in the target. For single crystal Si with a lattice spacing of $a=5.43 \AA$ we can see 


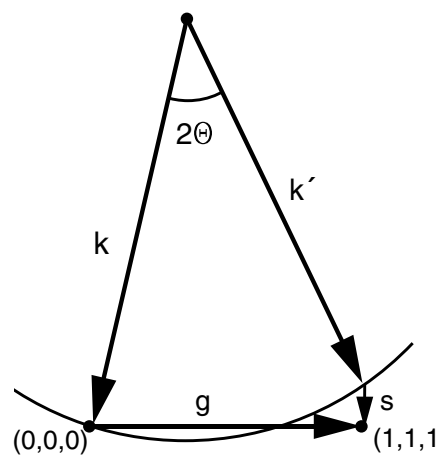

(a)

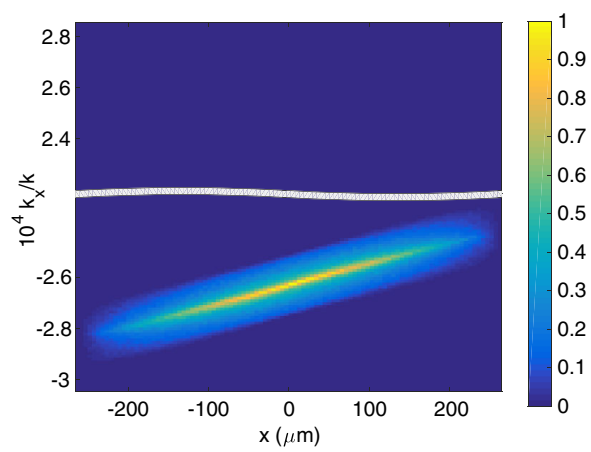

(b)

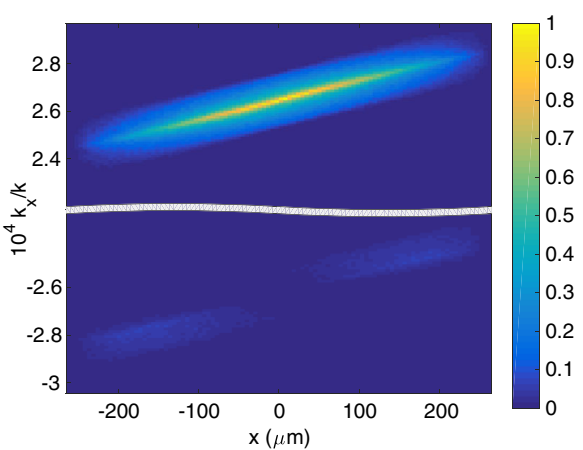

(c)

FIG. 2. (a) Scattering geometry for an incident particle. (b) The phase space of the incident electron bunch immediately prior to the target. (c) The calculated diffraction for the electron bunch from the rf photo-injector plotted as the relative density of electrons in the transverse phase space. With the thickness of the single crystal Si at $t=\xi / 2,98 \%$ of the beam undergoes one scattering event (upper panel). The remaining electrons (lower panel) did not scatter.

from Bragg's law, $\lambda=2 d_{\mathrm{hkl}} \sin \theta$ where $\lambda$ is the electron wavelength $\left(1.66 \times 10^{-3} \AA\right.$ at $\left.7 \mathrm{MeV}\right)$ and $d_{\mathrm{hkl}}$ are the interplanar spacings, that for the lowest order diffracted beam $[\mathrm{hkl}=(111)] 2 \theta=0.528 \mathrm{mrad}$ with respect to the incident electron beam. This small diffraction angle proves advantageous as it limits aberrations in the downstream electron optics. In the two-beam approximation the sample thickness, $t$, required for relativistic electrons and the (111) Bragg peak is given by the normalized amplitude $|\varphi|^{2}=\sin ^{2}\left(\frac{\pi t}{\xi}\right)$, where $\xi=100 \mathrm{~nm}$ is the extinction length given by $\xi=\pi \sqrt{1-\beta^{2}} V_{c} / \lambda F[23,24]$ with a structure factor of $F=22.6 \AA$ and $V_{c}=(0.543 \mathrm{~nm})^{3}$.

The scattering geometry is shown in Fig. 2(a) with $\vec{k}+\vec{g}=\vec{k}+\vec{s}$, where $k$ is the momentum vector for the incident electron, $k^{\prime}$ is the momentum of a diffracted electron, $g=2 \pi / d_{(111)}$ is the reciprocal lattice vector and $s$ is the deviation vector. Due to the finite emittance of the electron bunch, it is not possible for all the $k$ vectors of the incident particles to be properly aligned with the crystal plane, resulting in a a decreased probability of interacting with the crystal lattice for increasing $s$.

For clarity we will only consider the two beam case for a target consisting of a uniform medium. In practice, higherorder scattering events will be present requiring adjustments to sample dimensions and collection optics (bright-field vs dark-field). Targets consisting of layered materials can also be considered to produce the desired beam.

The intensity of the diffracted beam is

$$
I=\frac{\sin \left(\pi t s_{\mathrm{eff}}\right)^{2}}{\xi^{2} s_{\mathrm{eff}}^{2}}
$$

where $s_{\text {eff }}=\sqrt{s^{2}+\xi^{-2}}$ and $I_{0}=1-I$ is the intensity of the forward beam [25]. Figure 2(b) depicts the electron bunch phase space incident on the target. The forward and diffracted beams for a uniform thickness single Si crystal are shown in Fig. 2(c). These calculations are validated by electron diffraction experiments [26-28] using electron bunches at $\mathrm{MeV}$ energies with comparable emittances to these simulation parameters and single crystal silicon membranes that have demonstrated the near total scattering of an electron bunch into a single Bragg peak.

\section{DIFFRACTION CONTRAST MODULATION}

One approach to producing modulation in the electron bunch is to use a grating, Fig. 3(a). From (2) we can see that varying the thickness, $t$, in $x$ results in spatially alternating intensities in the transverse dimension for $I_{0}$ and $I$, i.e. diffraction contrast. Note that in this approach, the electrons are transmitted through the grating, and the grating is producing contrast due to the difference in diffracted intensity depending on the thickness of the grooves and ridges, but the diffraction itself is due to the $\mathrm{Si}$ atomic structure rather than the grating. The limitation on this modulation period is set by the minimum feature size that can be fabricated, however note that the image produced by the diffracted electron beam may be significantly demagnified to reach shorter periods. Subsequently, one of the two beams $\left(I_{0}, I\right)$ could be blocked and the remaining beam would be sent through emittance exchange (EEX) optics [29-33] to transfer the modulation from the transverse dimension to the longitudinal dimension.

The collective quality of the modulated electron bunch is determined with the bunching factor, which is a useful tool to measure how well phased the modulation is at a particular wavelength. The bunching factor is defined as

$$
b_{0 x}=\frac{1}{N_{e}} \sum_{p=1}^{N_{e}} e^{i k x_{p}}
$$

where $N_{e}$ is the number of electrons, $x_{p}$ is the transverse position of the $p^{\text {th }}$ particle, $k=2 \pi / \lambda_{x}$, and $\lambda_{x}$ is the period 


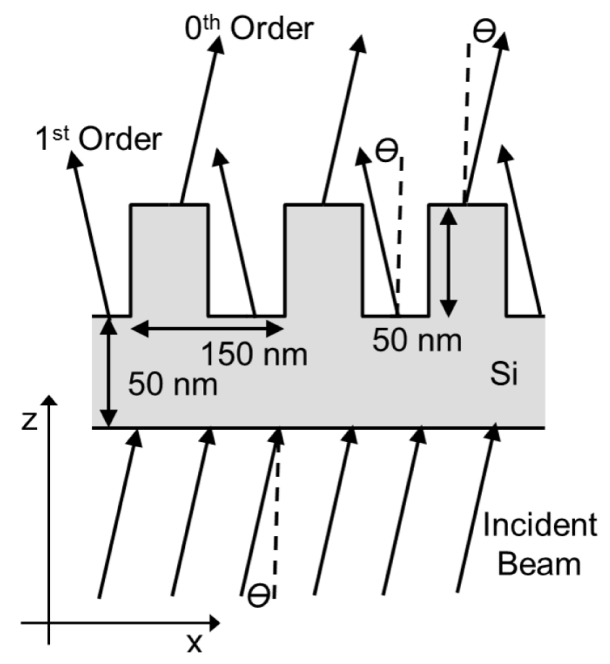

(a)

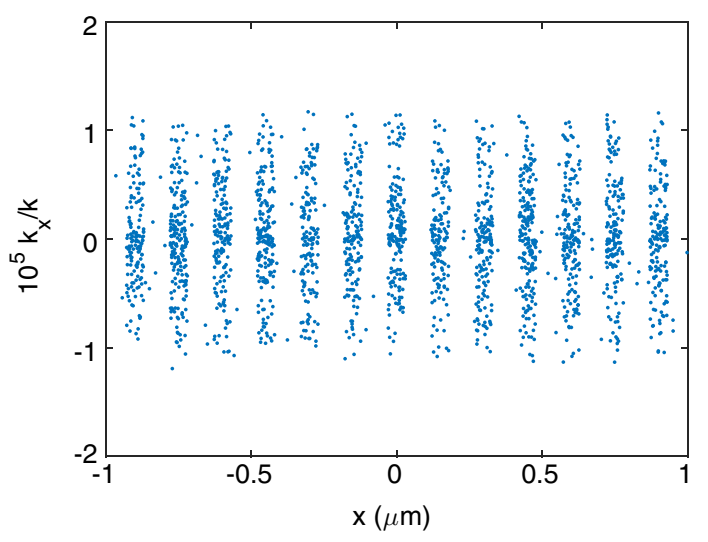

(b)

FIG. 3. (a) The single crystal Si grating. (b) The forward scattered (0th order) electron beam (with mean transverse momentum equal to the Bragg angle removed).

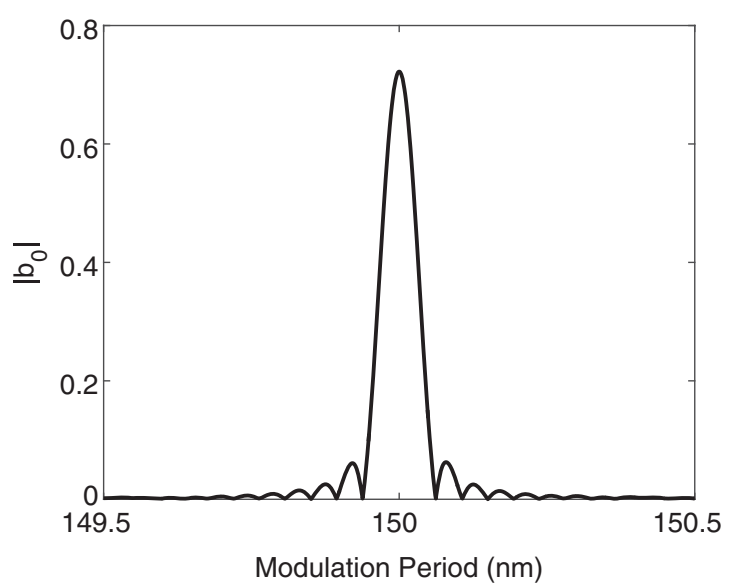

FIG. 4. The calculated $b_{0}$ with $\sim 150 \mathrm{~nm}$ spacing for the forward scattered (0th order) electron beam.

of modulation. For a random assortment of particles (no modulation) $b_{0 x}=1 / \sqrt{N_{e}} \ll 1$. For the modeled case with a grating period of $150 \mathrm{~nm}$, the forward scattered beam [Fig. 3(b)] contains $0.35 \mathrm{pC}$ and has a bunching factor $\left|b_{0 x}\right|=0.71$ (Fig. 4). The transverse modulation of either beam undergoes demagnification which can be varied and then is imaged into the longitudinal dimension via EEX. We have recently shown [34] that emittances with ratios as high as $10^{4}$ between longitudinal and transverse dimensions can be fully exchanged. If both beams are imaged without aberrations the modulation would disappear.

\section{NANOMETER-SCALE LONGITUDINAL MODULATION}

With an EEX beamline the emittance (phase-space) of an electron bunch in two dimensions is fully coupled and exchanged. For the beamline geometry we consider, the EEX line consists of two doglegs separated by a transverse deflecting cavity. With the modulation from the electron diffraction in the $\mathrm{x}$-dimension, the EEX beamline is arranged such that EEX occurs between the $\mathrm{x}-\mathrm{z}$ dimensions only; ideally the y-dimension is uncoupled. The behavior of the EEX beamline can be described by a transfer matrix $R$ applied to each electron's phase-space coordinates (shown without the y-dimension for simplicity)

$$
X^{\mathrm{T}}=\left(\begin{array}{llll}
x & x^{\prime} & z & \Delta p / p
\end{array}\right)
$$

where all terms are defined with respect to a reference particle, $x$ is the transverse coordinates of the particle, $x^{\prime}$ is the transverse angular divergence, $z=\beta c t$ is the longitudinal position, $p=\beta \gamma m_{e} c$ is the momentum, $c$ is the speed of light, $\beta=v / c, v$ is the electron velocity, $m_{e}$ is the electron mass, and $\gamma=1 / \sqrt{\left(1-\beta^{2}\right)}$ is the Lorentz factor. With the beam line arranged for complete emittance exchange the transfer matrix $R$ is

$$
R=\left(\begin{array}{cccc}
0 & 0 & -\frac{L}{\eta} & \eta-\frac{L \zeta}{\eta} \\
0 & 0 & -\frac{1}{\eta} & -\frac{\zeta}{\eta} \\
-\frac{\zeta}{\eta} & \eta-\frac{\zeta L}{\eta} & 0 & 0 \\
-\frac{1}{\eta} & -\frac{L}{\eta} & 0 & 0
\end{array}\right)
$$

where $\zeta$ is the longitudinal dispersion, $\eta$ is the horizontal dispersion, and $L$ is the drift length [34]. This transfer matrix will transfer the modulation from the transverse dimension into the longitudinal dimension.

The full accelerator setup is shown in Fig. 1 including the interaction area where coherent $\mathrm{x}$-rays would be produced 


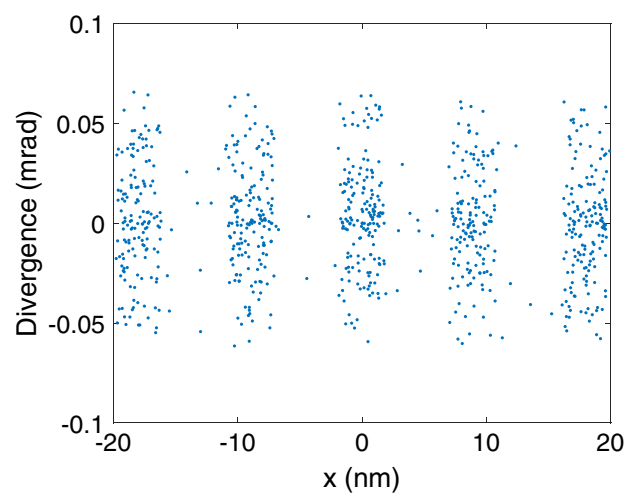

(a)

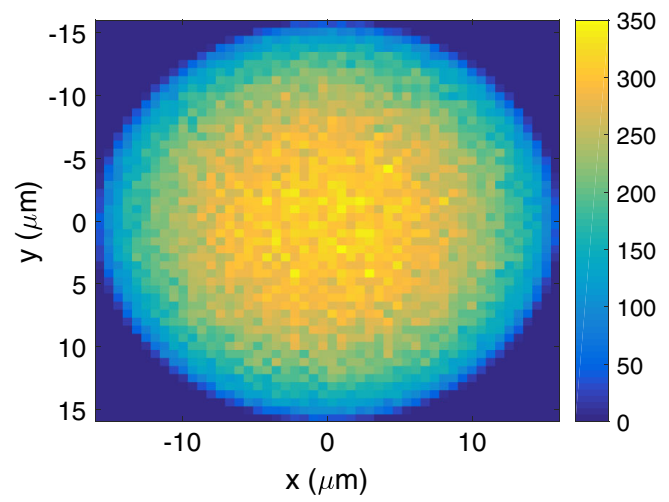

(b)

FIG. 5. (a) Individual electrons imaged after magnification and acceleration at the entrance of the EEX line. (b) Full transverse density profile of the electron bunch visualized as a two dimensional histogram of particle count in the simulation.

via inverse Compton scattering (ICS) of a high power laser or $\mathrm{THz}$ field on the modulated electrons. A test case was analyzed for diffraction from a grating with $150 \mathrm{~nm}$ period and demagnification factor of $1 / 120$ to produce a $1.24 \mathrm{~nm}$ modulation period which would produce coherent $1 \mathrm{keV}$ X-rays. Diffraction contrast modulation with the Si structure and dimensions shown in Fig. 3(a) was used. After interacting with the Si grating the forward scattered beam is accelerated in a short $\mathrm{x}$-band linac from $7 \mathrm{MeV}$ to 22.5 MeV. The accelerator is described in [35] and the arrangement of the EEX line is given in $[34,36]$. The imaging quadrupole lenses are set up as a telescope that demagnifies the beam by a factor of $1 / 14$, and then the EEX line further demagnifies by a factor of $1 / 6$. These demagnification factors in combination with the modest demagnification due to acceleration result in a $1.24 \mathrm{~nm}$ modulation at the EEX output. Fig. 5(a) shows the imaged pattern of the $\mathrm{Si}$ grating after being accelerated and magnified with $b_{0 x}=0.61$ for the entire electron bunch. The full transverse density profile of the electron bunch is shown in Fig. 5(b). The bunching factor calculated locally as a function of transverse coordinates is shown in Fig. 6(a)

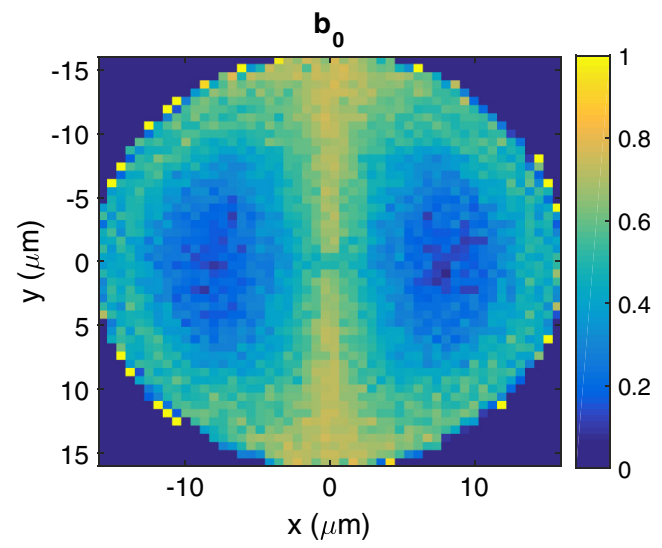

(a) along with the relative phase between the Fig. 6(b) electron beam modulation showing a flat phase profile across the full bunch.

In practice emittance exchange suffers from some aberrations which can limit the ability of the EEX line to transfer the modulation into the longitudinal dimension. In [34] an aberration corrected geometry for the EEX line was found to have a transfer matrix with aberration-free performance with emittances differing by four orders of magnitude between the beamlet produced by each grating period and the longitudinal bunch. The aberration corrected geometry required the addition of only three sextupoles and one octupole. The analytical and numerical performance from PARMELA simulations of the EEX line is given in Table I. The transfer matrix contains some residual selfcoupling for both the transverse and longitudinal dimension. The electron bunch is primarily sensitive to residual self-coupling in the longitudinal dimension, because the emittance of each beamlet produced by individual grating periods must be exchanged with the full emittance of the longitudinal bunch. As a result, the performance of the EEX line was optimized to minimize the residual

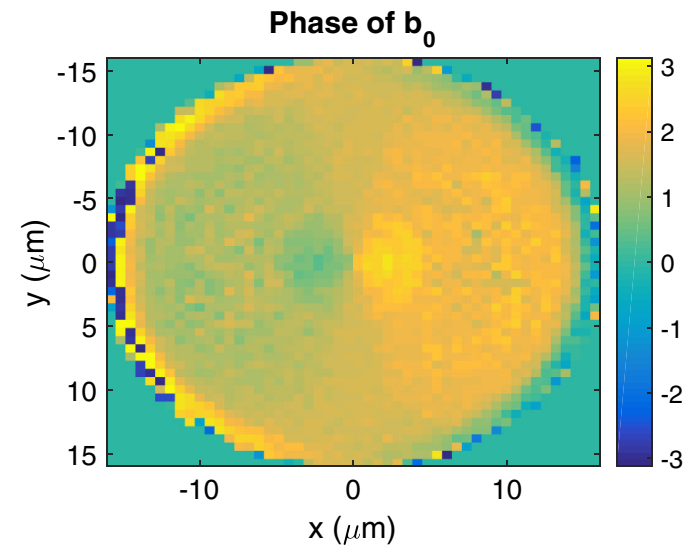

(b)

FIG. 6. (a) The bunching factor (b) and the relative phase in radians as a function of the transverse position in the beam. 
TABLE I. First order transfer matrix.

\begin{tabular}{lccccccccc}
\hline \hline Element & Symbol & \multicolumn{1}{c}{ Analytical } \\
\hline EEX Beamline & $R$ & $\left(\begin{array}{ccccccccc}0 & 0 & -7.27 & -0.0530 \\
& & 0 & -5.27 & -0.176 \\
0 & 0.176 & -0.043 & 0 & 0 \\
-5.27 & -6.97 & 0 & 0\end{array}\right) \quad\left(\begin{array}{ccccc}-1.13 \times 10^{-3} & -7.58 \times 10^{-4} & -6.92 & -0.034 \\
-5.80 \times 10^{-3} & -1.05 \times 10^{-3} & -5.27 & -0.155 \\
-0.174 & -0.029 & -3.72 \times 10^{-6} & -1.54 \times 10^{-5} \\
-5.33 & -6.63 & 1.72 \times 10^{-3} & -3.69 \times 10^{-4}\end{array}\right)$ \\
\hline \hline
\end{tabular}

self-coupling in the longitudinal dimension as this dimension will contain the small final emittance.

In addition to the minimization of the residual selfcoupling, a low longitudinal emittance is beneficial to the EEX process. The electron bunch longitudinal emittance prior to the EEX line is $\epsilon_{z n}=5 \mathrm{~nm}$-rad, as shown in Fig. 8(a). Some emittance growth is observed with the exchange of the longitudinal phase space into the transverse phase space with a doubling of the emittance in the final transverse dimension to $\epsilon_{x n}=10 \mathrm{~nm}-\mathrm{rad}$. This increase in emittance does not adversely impact the production of $\mathrm{X}$-rays as discussed in Sec. V.

In Fig. 7(a) the final bunching factor of the electron bunch after EEX is shown vs the modulation period. The bunching factor has decreased from a $b_{0 x}=0.61$ at the entrance of the EEX line to $b_{0 z}=0.14$ due to residual selfcoupling of the longitudinal phase space. However, the line width of Fig. 7(a) is $0.01 \AA$ which corresponds to a coherent content equivalent to the transform limited bandwidth of the electron bunch after the grating. This indicates

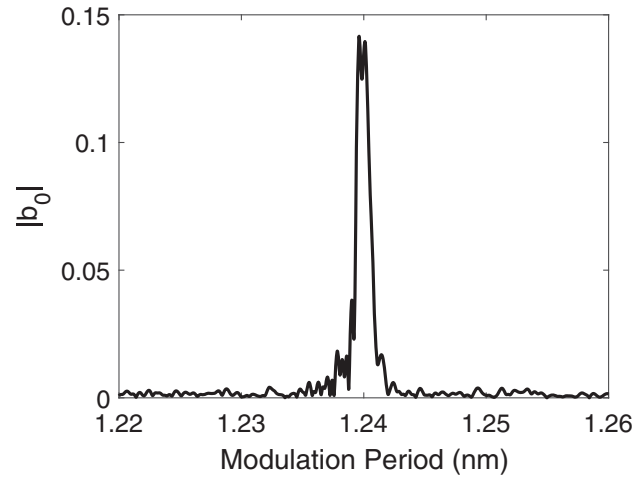

(a)

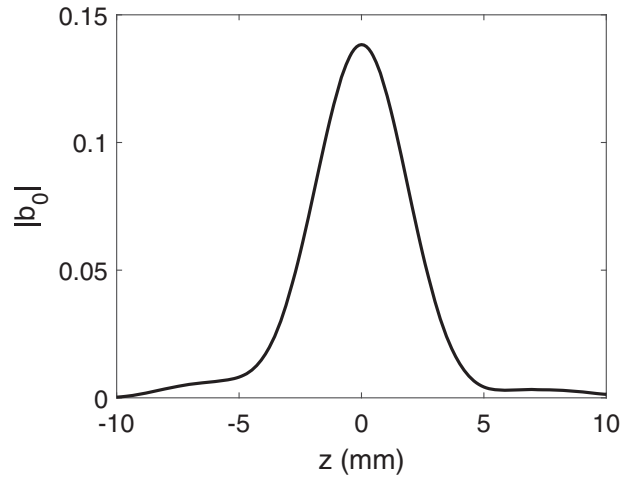

(b)

FIG. 7. (a) Individual electrons imaged after demagnification and acceleration at the entrance of the EEX line. (b) The bunching factor as a function of the transverse position in the beam.

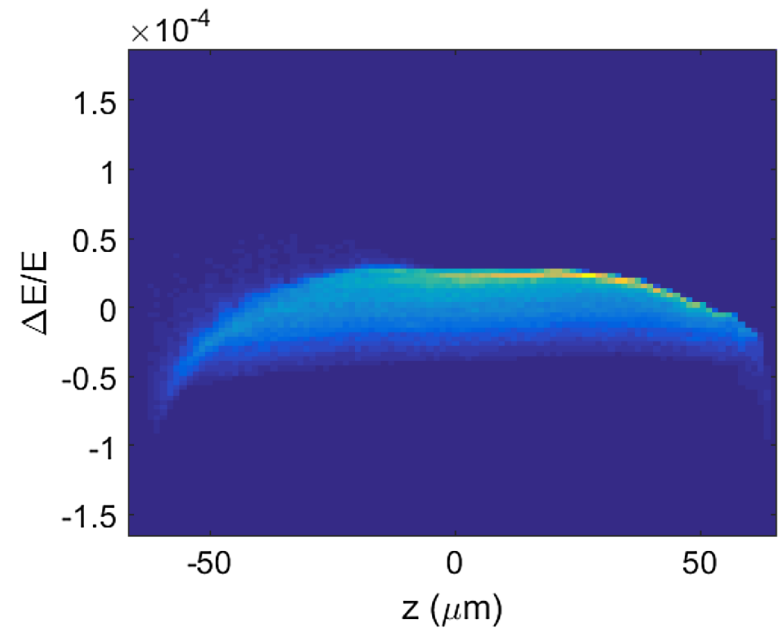

(a)

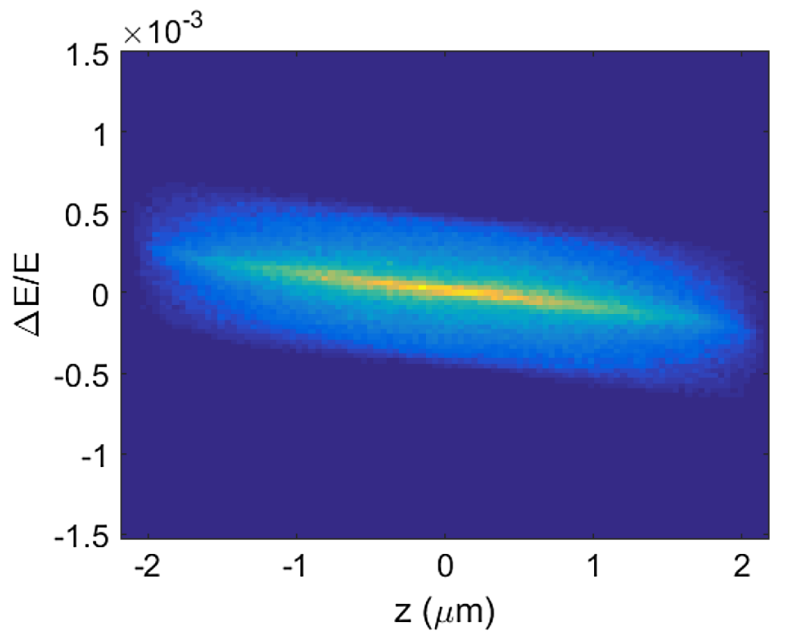

(b)

FIG. 8. The relative density of the electron bunch for the longitudinal phase space (a) before and (b) after emittance exchange. 


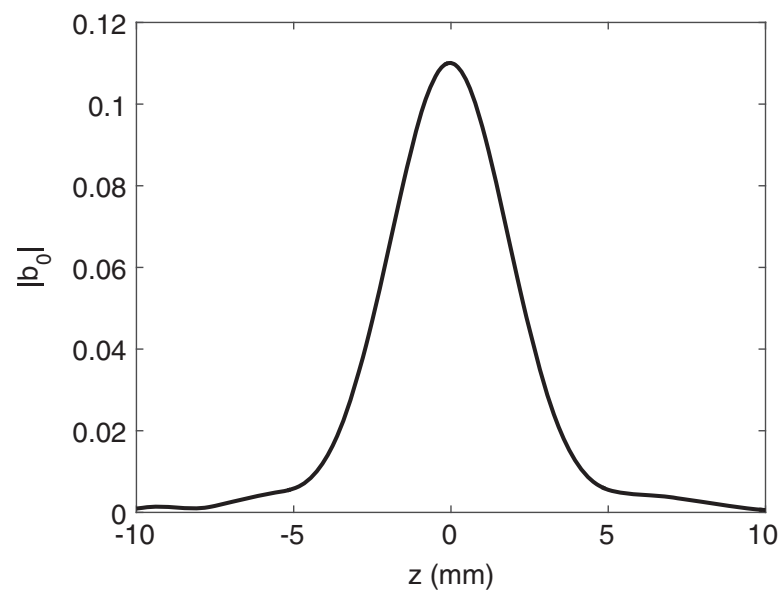

FIG. 9. The bunching factor for the electron bunch modulation at $1.24 \mathrm{~nm}$ after the EEX line. The depth of field is $4 \mathrm{~mm}$, approximately ten times the gain length or four times the nominal laser pulse length.

that the modulation in the electron bunch has been uniformly demagnified without impacting the coherence. The modulation as a function of position in the electron bunch is shown in distance is shown in Fig. 7(b) with a strong coherent content throughout the longitudinal extent of the electron bunch.

The impact of the emittance exchange line can also be observed in Fig. 8 with the longitudinal phase space shown before and after the EEX line. The global phase space shows that there is no correlation before and after emittance exchange. The electron bunch length is compressed by the EEX line resulting in an increase in the peak current from a few amps to $\sim 50 \mathrm{~A}$ at the exit of the EEX line. This compression is beneficial because the electron bunch travels from the grating through the EEX line with low current limiting the impact of space charge. The depth of field for the modulated electron bunch is also critical in determining its ability to interact with the laser undulator. The global bunching factor is shown in Fig. 9 as the electron bunch propagates after the EEX line. Due to the low energy the depth of field for the modulation is only $\sim 5 \mathrm{~mm}$, but this is sufficiently long to interact with the entire length of the laser undulator.

\section{X-RAY PERFORMANCE}

We can estimate the $1 \mathrm{keV}$ x-ray performance by treating the ICS interaction as an effective undulator field and applying the universal FEL scaling formulas of Xie [37]. The electron spot size at the crystal results in scattering from about 1800 grooves thus producing 1800 nanobunches after EEX for a total electron bunch length of $8 \mathrm{fs}$ with peak current $47 \mathrm{~A}$. The high-power IR laser pulse used for ICS is equivalent to a static undulator in this X-ray performance estimate. This is physically correct if the laser field is uniform, a challenging issue for which solutions
[38-40] have been proposed. For an IR laser with $10 \mu \mathrm{m}$ wavelength and strength parameter $a_{0}=0.4$, electron beam Twiss parameter $\beta_{x}=2 \mathrm{~mm}$, normalized transverse emittance $\epsilon_{x n}=10 \mathrm{~nm}$-rad, and energy spread $\Delta E / E=$ $2.5 \times 10^{-4}$, the effective Pierce parameter including the effects of emittance, diffraction, and energy spread [41] is $\rho=3.2 \times 10^{-4}$, the exponential gain length including these effects is $L_{g}=401 \mu \mathrm{m}$, and the saturated power is $810 \mathrm{~kW}$. Only 2-3 gain lengths are required to reach saturation because the electrons are bunched before interacting with the laser, allowing use of a few ps laser pulse. Furthermore because the energy spread is an order of magnitude smaller than the Pierce parameter, the saturation power could be substantially exceeded by using a chirped laser pulse equivalent to a tapered undulator.

\section{PHASE-CONTRAST MODULATION}

In order to extend the electron bunch modulation into the hard x-ray regime (i.e., sub-nm modulation) without significant amounts of demagnification, phase-contrast imaging of diffracted electrons can directly provide modulation on the order of the atomic structure spacing $(\sim 5 \AA)$. Phase-contrast imaging relies on the interference of the diffracted beam, $\phi$, with the forward scattered beam, $\phi_{0}$ :

$$
\begin{aligned}
\varphi_{0}(r) & =\varphi_{0}(z) e^{i \overrightarrow{k_{0} \cdot \vec{r}}} \\
\varphi(r) & =\varphi(z) e^{i\left(\overrightarrow{k_{0}}+\vec{g}\right) \cdot \vec{r}}
\end{aligned}
$$

The amplitude of these two wave functions is determined by the excitation of two Bloch waves $\left(\psi_{1}, \psi_{2}\right)$ at the entrance of the crystal and the relative phase of these two Bloch waves at the exit of the crystal. As the electron bunch arrives at the Si crystal, no modulation is present in the beam and its wave function is a plane wave with a flat phase front. Once the electron penetrates into the crystal it can no longer be described as a plane wave, because the $\mathrm{Si}$ atoms act as potential wells and apply a spatially varying phase advance. The Bloch waves $\left(\psi_{1}, \psi_{2}\right)$ are the new eigenstates for the electron, and the incident plane wave excites these two waves with equal amplitude for $\vec{s}=0$. Note that $\left(\psi_{1}, \psi_{2}\right)$ propagate colinearly, but they have unique wave vectors $\left(k^{(1)}, k^{(2)}\right)$ or $\left(\vec{k}+\gamma^{(1)} \hat{z}, \vec{k}+\gamma^{(2)} \hat{z}\right)$. When the electrons exit the crystal we once again can describe them as plane waves with modulated phases. However, depending on the relative phase and amplitude of the two Bloch waves, two diffracted plane waves can be excited $\left(\phi_{0}, \phi\right)$. At the crystal exit the two waves are

$$
\begin{aligned}
\phi_{0}(z)= & e^{i s z / 2}\left[\cos \left(\frac{s_{\mathrm{eff}} z}{2}\right)\right. \\
& \left.-i \cos \left(\cot (s \xi)^{-1}\right) \sin \left(\frac{s_{\mathrm{eff}} z}{2}\right)\right]
\end{aligned}
$$




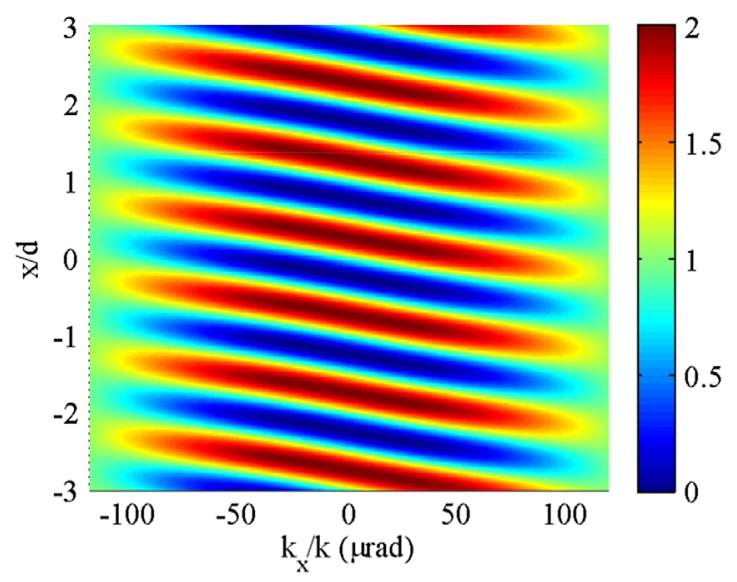

(a)

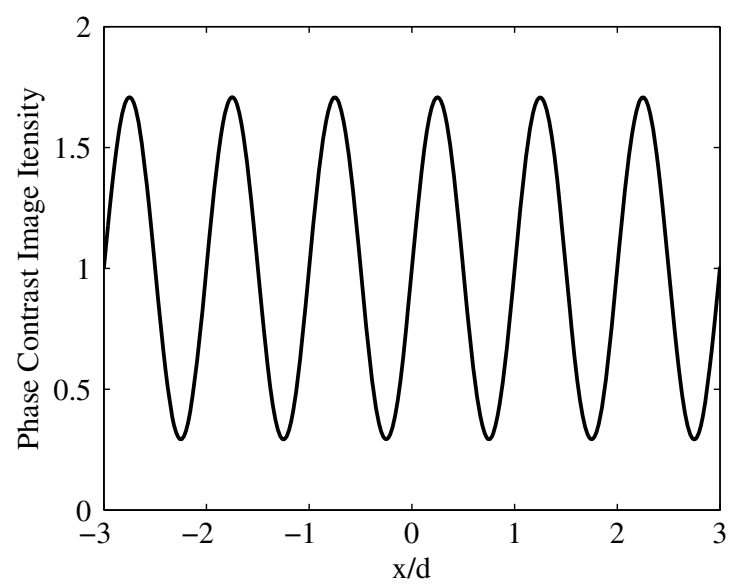

(b)

FIG. 10. (a) Phase-contrast modulation as a function of incident angle and transverse position. (b) Integrated phase-contrast modulation over the incident electron distribution $\left(\sigma_{x^{\prime}} \approx 50 \mu \mathrm{rad}\right)$ at the exit of the Si crystal.

$$
\phi(z)=i e^{i s z / 2} \sin \left(\cot (s \xi)^{-1}\right) \sin \left(\frac{s_{\mathrm{eff}} z}{2}\right)
$$

noting that in $\hat{x}$ both waves contain modulation in phase on the order of $g$. We can describe the total beam image as

$$
I_{\mathrm{tot}}=\left(\varphi_{0}+\varphi\right)\left(\varphi_{0}+\varphi\right)^{*},
$$

which becomes

$$
\begin{aligned}
I_{\mathrm{tot}}= & 1+2 \sin \left(\cot (s \xi)^{-1}\right) \sin \left(\frac{s_{\mathrm{eff}} z}{2}\right) \\
& \times\left[\sin (g x) \cos \left(\frac{s_{\mathrm{eff}} z}{2}\right)\right. \\
& \left.+\cos (g x) \cos \left(\cot (s \xi)^{-1}\right) \sin \left(\frac{s_{\mathrm{eff}} z}{2}\right)\right] .
\end{aligned}
$$

We evaluate this expression for a sample depth of $75 \mathrm{~nm}$ or $3 \xi / 4$ which results in the optimal mix $(50 / 50)$ of the forward and diffracted beam. Figure 10(a) is the intensity of the diffraction pattern in the transverse coordinate for varying incident angle and (b) is the accumulated population for our design case focused to a RMS spot size at the crystal of $\sigma_{x}=11.5 \mu \mathrm{m}$ with angular distribution $\sigma_{x^{\prime}} \approx 50 \mu \mathrm{rad}$ to reduce the number of modulation periods. Excellent phase contrast is observed at the exit of the Si crystal.

\section{ABERRATIONS}

Phase-contrast imaging has not yet been demonstrated with rf photo injectors, however the electron bunch produced by a state-of-the-art rf gun has sufficient beam quality as shown in the previous section. Imaging the transverse modulation at the interaction point will require careful analysis of aberrations from the optical elements in the setup. We estimate the effects of aberrations with analytical calculations that assume imaging with a standard objective lens. For phase contrast we must consider the wave-optical formulation of aberrations due to imaging. In the wave-optical formulation the effect of aberrations is given by a phase shift $W(\theta)=2 \pi \Delta s / \lambda$ where $\Delta s$ is the change in optical path with respect to the ideal spherical wave front and $\theta$ is the scattered angle. The phase shift can result from three effects: spherical aberrations, fluctuations in the thickness of the sample, or change in focal length due to energy. These effects combine to give a total phase shift (Eq. (3.65) in [42]) of

$$
W(\theta)=\frac{\pi}{2 \lambda}\left(C_{s} \theta^{4}-2(\Delta f-\Delta a) \theta^{2}\right)
$$

where $\Delta f=f \Delta E / E$ and $\Delta a$ is the variation of the longitudinal position of the sample (effectively due to tilt) and should be kept to on the order of $\Delta f$. It is sufficient to keep $\Delta a$ on the order of $40 \mathrm{~nm}$ (with an illumination spot of $11.5 \mu \mathrm{m}$ this is a tilt of $4 \mathrm{mrad}$ ), which is a weaker tolerance than the required $0.1 \mathrm{mrad}$ alignment for the crystal plane.

Observing modulation of the electron beam that is on the order of the lattice spacing $a=5.43 \AA$ requires the ability to collect electrons from a transverse momentum space that covers $k_{\perp}=4 \pi / a$ or $k_{\perp} / k_{0}=0.5 \mathrm{mrad}$ which includes a minimum of two diffraction peaks. To analyze the imaged beam we take the amplitude distribution at the output of the crystal $\phi\left(r, z_{o}\right)=\phi_{0}\left(r, z_{o}\right)+\phi\left(r, z_{o}\right)$ and propagate it as spherical wave fronts to the image location [42] including aberrations from (12). In the absence of aberrations, the objective lens will reimage the beam such that the relative accumulated phase at the image plane $\left(z_{\mathrm{i}}\right)$ is zero, recreating the image

$$
\phi_{i}\left(r, z_{\mathrm{i}}\right)=\frac{1}{M} \iint F(\mathrm{q}) e^{i 2 \pi \mathrm{q} \cdot \mathrm{r}} \mathrm{d}^{2} \mathrm{q}=\frac{1}{M} \phi\left(r, z_{\mathrm{o}}\right)
$$

where $F(\mathrm{q})=\int_{S} \phi_{S}(\mathrm{r}) e^{-i 2 \pi \mathrm{q} \cdot \mathrm{r}} \mathrm{d}^{2} \mathrm{r}$ and $q=k_{\perp} / k_{0} \lambda ;=\theta / \lambda$ is the transverse momentum. Aberrations given by the 


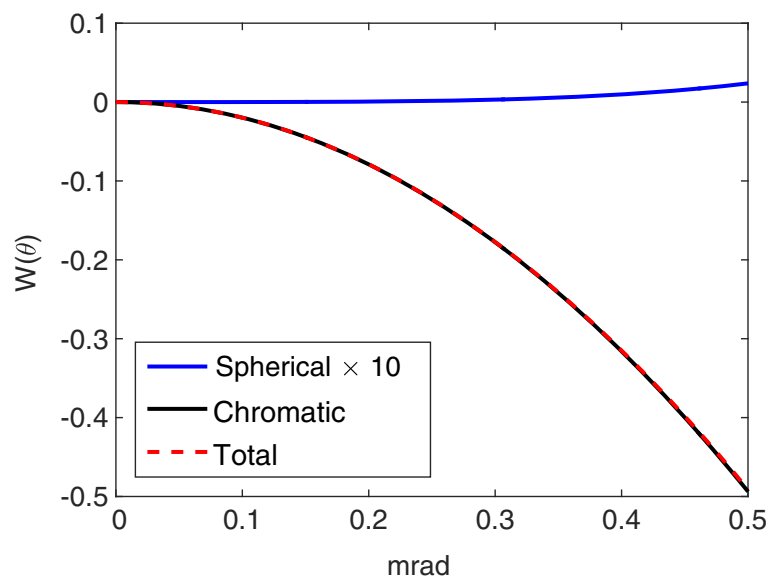

(a)

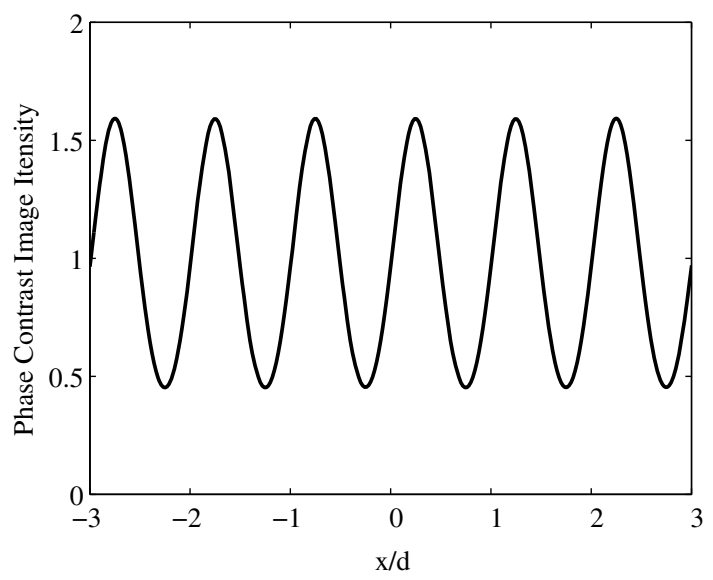

(b)

FIG. 11. (a) Added phase as a function of the angle of divergence for a focal length of $4 \mathrm{~mm}$ and a $\Delta E / E=1.3 \times 10^{-5}$. (b) Electron population in phase-contrast image propagated through a lens with a focal length of $4 \mathrm{~mm}$ for the initial conditions in Fig. 10(b).

momentum space pupil function $H(\theta)=e^{-i W(\theta)} M(\theta)$ are included where the diaphragm opening $M(\theta)$ is a step function describing the angle of rays which are collected for the image. The new imaging formulation is

$$
\phi_{i}\left(r, z_{\mathrm{i}}\right)=\frac{1}{M} \iint F(\mathrm{q}) e^{i 2 \pi \mathrm{q} \cdot \mathrm{r}} \mathrm{H}(\mathrm{q}) \mathrm{d}^{2} \mathrm{q},
$$

which can also be described as a convolution of the source image with the pupil function for the objective lens $h(r)=$ $\mathrm{F}^{-1}\{H(\theta)\}$, i.e., the inverse Fourier transform of the momentum space pupil function. The amplitude distribution of the reimaged electron beam is

$$
\phi_{i}\left(r, z_{\text {image }}\right)=\frac{1}{M} \phi\left(r, z_{\mathrm{o}}\right) \otimes h(r)
$$

The aberrations and imaged electron beam are shown in Fig. 11 assuming that the sample is placed a distance $S_{1}=$ $2 f$ from the objective lens. Figure 11(b) shows that a strong modulation is possible for the given beam and transport conditions. While this represents a first step in the analysis of a feasible arrangement for phase-contrast imaging, a significant amount of work remains in assessing the impact of aberrations introduced by the accelerating structures and the emittance exchange line. This will require operation at lower emittance or the addition of additional magnetic optics to reduce the impact of aberrations.

\section{CONCLUSIONS}

In conclusion, we have presented detailed calculations showing how to produce a moderately relativistic electron beam that is coherently modulated at a nanometer scale in preparation for generating fully coherent $\mathrm{x}$-rays. Furthermore, we have presented a method to extend the technique to sub-nanometer modulation using phase contrast electron diffraction that would enable generation of coherent hard $\mathrm{x}$-rays. Using conventional ICS from a laser pulse, electron beams prepared in this manner can be a stable source of powerful fully coherent x-rays from a table-top source. Such a source would have many impacts, enabling labs and groups around the world access at modest cost to the remarkable science produced by ultrashort pulse coherent $\mathrm{x}$-rays. The output pulse energy is modest due to the low charge employed, yet the temporal coherence and resulting spectral purity are likely to open new applications that are not achievable with SASE-based XFELs. The coherent power significantly exceeds the startup noise in a SASE FEL and could be a useful seed source to transfer full coherence and improved stability to the x-ray beams produced large facilities.

\section{ACKNOWLEDGMENTS}

The authors gratefully acknowledge Lucas Malin, John Spence and Philippe Piot for many useful discussions. This work was supported by NSF Grant No. DMR-1042342 and 1632780, DOE Grant No. DE-FG02-10ER46745 and DE-AC02-76SF00515, and DARPA Grant No. N6600111-1-4192.

[1] P. Emma, R. Akre, J. Arthur, R. Bionta, C. Bostedt, J. Bozek, A. Brachmann, P. Bucksbaum, R. Coffee, F.-J. Decker et al., First lasing and operation of an ångstromwavelength free-electron laser, Nat. Photonics 4, 641 (2010).

[2] D. Pile, X-rays: First light from SACLA, Nat. Photonics 5, 456 (2011).

[3] M. Altarelli, R. Brinkmann, M. Chergui, W. Decking, B. Dobson, S. Düsterer, G. Grübel, W. Graeff, H. Graafsma, J. Hajdu et al., Technical Design Report No. DESY 2006097, 2006, http://xfel.desy.de/localfsExplorer_read? 
currentPath=/afs/desy.de/group/xfel/wof/EPT/TDR/XFELTDR-final.pdf.

[4] J. Feldhaus, E. Saldin, J. Schneider, E. Schneidmiller, and M. Yurkov, Possible application of X-ray optical elements for reducing the spectral bandwidth of an X-ray SASE FEL, Opt. Commun. 140, 341 (1997).

[5] E. Saldin, E. Schneidmiller, Y. V. Shvyd'ko, and M. Yurkov, X-ray FEL with a meV bandwidth, Nucl. Instrum. Methods Phys. Res., Sect. A 475, 357 (2001).

[6] Y. Ding, Z. Huang, and R. D. Ruth, Two-bunch selfseeding for narrow-bandwidth hard $\mathrm{x}$-ray free-electron lasers, Phys. Rev. ST Accel. Beams 13, 060703 (2010).

[7] I. Ben-Zvi, L. Di Mauro, S. Krinsky, M. White, and L. Yu, Proposed UV FEL user facility at BNL, Nucl. Instrum. Methods Phys. Res., Sect. A 304, 181 (1991).

[8] L. H. Yu, Generation of intense uv radiation by subharmonically seeded single-pass free-electron lasers, Phys. Rev. A 44, 5178 (1991).

[9] L.-H. Yu, M. Babzien, I. Ben-Zvi, L. DiMauro, A. Doyuran, W. Graves, E. Johnson, S. Krinsky, R. Malone, I. Pogorelsky et al., High-gain harmonic-generation freeelectron laser, Science 289, 932 (2000).

[10] D. Xiang and G. Stupakov, Echo-enabled harmonic generation free electron laser, Phys. Rev. ST Accel. Beams 12, 030702 (2009).

[11] Z. Zhao, D. Wang, J. Chen, Z. Chen, H. Deng, J. Ding, C. Feng, Q. Gu, M. Huang, T. Lan et al., First lasing of an echo-enabled harmonic generation free-electron laser, Nat. Photonics 6, 360 (2012).

[12] W. Graves, F. Kärtner, D. Moncton, and P. Piot, Intense Superradiant X Rays from a Compact Source Using a Nanocathode Array and Emittance Exchange, Phys. Rev. Lett. 108, 263904 (2012).

[13] W. Graves, K. Berggren, S. Carbajo, R. Hobbs, K. Hong, W. Huang, F. Kärtner, P. Keathley, D. Moncton, E. Nanni et al., in Proceedings of the 2013 FEL Conference, New York, USA (2013), http://accelconf.web.cern.ch/ AccelConf/FEL2013/papers/wepso24.pdf.

[14] Y. Murooka, N. Naruse, S. Sakakihara, M. Ishimaru, J. Yang, and K. Tanimura, Transmission-electron diffraction by $\mathrm{MeV}$ electron pulses, Appl. Phys. Lett. 98, 251903 (2011).

[15] J. Hastings, F. Rudakov, D. Dowell, J. Schmerge, J. Cardoza, J. Castro, S. Gierman, H. Loos, and P. Weber, Ultrafast time-resolved electron diffraction with megavolt electron beams, Appl. Phys. Lett. 89, 184109 (2006).

[16] P. Musumeci, J. Moody, and C. Scoby, Relativistic electron diffraction at the UCLA Pegasus photoinjector laboratory, Ultramicroscopy 108, 1450 (2008).

[17] M. Hada, J. Hirscht, D. Zhang, S. Manz, K. Pichugin, D. Mazurenko, S. Bayesteh, H. Delsim-Hashemi, K. Floettmann, M. Huening et al., REGAE: New Source for Atomically Resolved Dynamics, in International Conference on Ultrafast Structural Dynamics (Optical Society of America, 2012), paper JT2A.47.

[18] R. D. Miller, Annu. Rev. Phys. Chem. 65, 583 (2014).

[19] L. Young and J. Billen, Technical Report No. LA-UR-961835, 2003.

[20] O. Luiten, S. Van der Geer, M. De Loos, F. Kiewiet, and M. Van Der Wiel, How to Realize Uniform
Three-Dimensional Ellipsoidal Electron Bunches, Phys. Rev. Lett. 93, 094802 (2004).

[21] P. Musumeci, J. T. Moody, R. J. England, J. B. Rosenzweig, and T. Tran, Experimental Generation and Characterization of Uniformly Filled Ellipsoidal Electron-Beam Distributions, Phys. Rev. Lett. 100, 244801 (2008).

[22] T. Li, B. L. Rickman, and W. A. Schroeder, Emission properties of body-centered cubic elemental metal photocathodes, J. Appl. Phys. 117, 134901 (2015).

[23] B. Fultz and J. M. Howe, Transmission Electron Microscopy and Diffractometry of Materials (Springer Science \& Business Media, New York, 2012).

[24] P. B. Hirsch, A. Howie, R. Nicholson, D. Pashley, and M. J. Whelan, Electron Microscopy of Thin Crystals (Butterworth Inc., Washington, D.C., 1966).

[25] A. Howie, Diffraction channelling of fast electrons and positrons in crystals, Philos. Mag. 14, 223 (1966).

[26] E. Nanni, W. Graves, R. Kirian, R. Li, C. Limborg, X. Shen, J. Spence, S. Weathersby, and U. Weierstall, in 7th International Particle Accelerator Conference (IPAC'16), Busan, Korea, 2016 (JACOW, Geneva, Switzerland, 2016), p. 1611 .

[27] L. Malin et al., in Proc. of Int. Particle Accel. Conf (IPAC2017) (Copenhagen, Denmark, 2017), p. THPAB088, ISBN 978-3-95450-147-2.

[28] C. Zhang et al., in Proc. of 2017 Int'l Free Electron Laser Conference (FEL2017) (Santa Fe, USA, 2017), p. TUP038, ISBN 978-3-95450-147-2, http://fel2017.jacow.de/papers/ tup038.pdf.

[29] M. Cornacchia and P. Emma, Transverse to longitudinal emittance exchange, Phys. Rev. ST Accel. Beams 5, 084001 (2002).

[30] P. Emma, Z. Huang, K.-J. Kim, and P. Piot, Transverse-tolongitudinal emittance exchange to improve performance of high-gain free-electron lasers, Phys. Rev. ST Accel. Beams 9, 100702 (2006).

[31] Y.-E. Sun, P. Piot, A. Johnson, A. Lumpkin, T. Maxwell, J. Ruan, and R. Thurman-Keup, Tunable Subpicosecond Electron-Bunch-Train Generation Using a TransverseTo-Longitudinal Phase-Space Exchange Technique, Phys. Rev. Lett. 105, 234801 (2010).

[32] B. E. Carlsten, K. A. Bishofberger, S. J. Russell, and N. A. Yampolsky, Using an emittance exchanger as a bunch compressor, Phys. Rev. ST Accel. Beams 14, 084403 (2011).

[33] D. Xiang and A. Chao, Emittance and phase space exchange for advanced beam manipulation and diagnostics, Phys. Rev. ST Accel. Beams 14, 114001 (2011).

[34] E. A. Nanni and W. S. Graves, Aberration corrected emittance exchange, Phys. Rev. ST Accel. Beams 18, 084401 (2015).

[35] W. Graves, J. Bessuille, P. Brown, S. Carbajo, V. Dolgashev, K.-H. Hong, E. Ihloff, B. Khaykovich, H. Lin, K. Murari et al., Compact x-ray source based on burst-mode inverse Compton scattering at $100 \mathrm{kHz}$, Phys. Rev. ST Accel. Beams 17, 120701 (2014).

[36] E. Nanni, W. Graves, and P. Piot, in 2014 International Particle Accelerator Conference (JACoW, Geneva, 2014), pp. 1952-1955. 
[37] M. Xie, Exact and variational solutions of 3D eigenmodes in high gain FELs, Nucl. Instrum. Methods Phys. Res., Sect. A 445, 59 (2000).

[38] C. Chang, J. Liang, D. Hei, M. F. Becker, K. Tang, Y. Feng, V. Yakimenko, C. Pellegrini, and J. Wu, High-brightness $\mathrm{X}$-ray free-electron laser with an optical undulator by pulse shaping, Opt. Express 21, 32013 (2013).

[39] D. Seipt, S. G. Rykovanov, A. Surzhykov, and S. Fritzsche, Narrowband inverse Compton scattering X-ray sources at high laser intensities, Phys. Rev. A 91, 033402 (2015).
[40] J. E. Lawler, J. Bisognano, R. A. Bosch, T. C. Chiang, M. A. Green, K. Jacobs, T. Miller, R. Wehlitz, D. Yavuz, and R. C. York, Nearly copropagating sheared laser pulse FEL undulator for soft X-rays, J. Phys. D 46, 325501 (2013).

[41] R. Bonifacio, C. Pellegrini, and N. L, Collective instabilities and high-gain regime in a free electron laser, Opt. Commun. 50, 373 (1984).

[42] L. Reimer and H. Kohl, Transmission Electron Microscopy: Physics of Image Formation (Springer Science \& Business Media, New York, 2008), Vol. 36. 